\title{
Employing Orphan Nodes to Avoid Energy Holes in Wireless Sensor Networks
}

\author{
Sepideh Zareei ${ }^{1}$, Elham Babaee ${ }^{1}$, Rosli Salleh ${ }^{1}$, Saeed Moghadam ${ }^{2}$ \\ ${ }^{1}$ Faculty of Computer Science and Information Technology, University of Malaya, Kuala Lumpur, Malaysia \\ ${ }^{2}$ Faculty of Electrical, IT, and Computer Sciences, Qazvin Islamic Azad University, Qazvin, Iran \\ Email: s.zareei@siswa.um.edu.my, s.moghadam@qiau.ac.ir
}

Received May 2013

\begin{abstract}
When energy consumption by wireless sensor nodes gets off balance, partitions in the network appear because several of the nodes stop functioning. The respective network's lifetime also diminishes. This problem is commonly known as the "hot spot" or "energy hole" phenomenon. To resolve this issue, a Multi-Hop Decentralized Cluster-Based Routing (MDCR) protocol is proposed. This algorithm uses orphan nodes as intermediate nodes to form inter-cluster multi-hop routing and balance the energy consumption among sensor nodes. Simulation experiments have shown that MDCR is significantly better at prolonging network lifetime compared to the Adaptive Decentralized Re-Clustering Protocol.
\end{abstract}

Keywords: Wireless Sensor Networks; Clustering; Energy Efficiency; Orphan Nodes

\section{Introduction}

A wireless sensor network (WSN) consists of a large number of distributed sensor nodes. The capability to interact directly with physical phenomena have led to the deployment of a vast number of applications for WSNs including surveillance, machine failure diagnosis and chemical/biological detection [1,2].

Clustering approach involves grouping nodes into clusters; every cluster has a cluster head (CH) that acts as the cluster coordinator, while the remaining nodes are called cluster members (CM) [3]. In order to achieve equilibrium in the energy consumption between members, the role of $\mathrm{CH}$ is rotated throughout the CMs. Another issue that remains to be addressed is the variation in energy dissipation caused by the distance to the base stations. CHs which are further away from the base station use more energy in inter-cluster single-hop routing because the energy load is higher in long-range communication compared to closer CHs. On the other hand, in inter-cluster multi-hop routing, $\mathrm{CHs}$ near the base station are used as intermediate nodes for transmitting data to the base station. Therefore, these nodes forward more data and rapidly dissipate their energy. The disparity in energy consumption by nodes results in an energy hole near the base station. Hence, designing an energy-efficient routing protocol, which also maintains the energy balance in the network, is the researchers' primary concern.

In this paper, Multi-Hop Decentralized Cluster-Based
Routing is proposed, which is an extension of the Adaptive Decentralized Re-Clustering protocol. Rather than employing $\mathrm{CHs}$ to form inter-cluster multi-hop routing, MDCR makes use of orphan nodes as intermediate nodes for multi-hop routing. By using this method, $\mathrm{CHs}$ located close to the base station preserve their energy for coordinating their cluster. Thus, energy consumption can be balanced among CHs. In addition, MDCR addresses the problem of inefficient clustering in ADRP by selecting future CHs located near the cluster's center.

The paper is arranged as follows. The related works in this area are covered in Section 2, the network model is presented in Section 3, while Section 4 deals with the orphan node problem and the solutions proposed to solve it. Section 5 explains in detail the Multi-Hop Decentralized Cluster-Based Routing protocol. Simulation results along with an analysis are described in Section 6 and the conclusions are presented in the final Section 7.

\section{Related Work}

LEACH [4] is a distributed single-hop clustering algorithm, in which every sensor node decides to be a $\mathrm{CH}$ or not, based on the following equation:

$$
P(n)= \begin{cases}\frac{k}{1-k^{*}\left(r \bmod \frac{1}{k}\right)} & n \in G \\ 0 & \text { otherwise }\end{cases}
$$


where $k$ is the predetermined percentage of CHs (e.g. $k=0.05$ ), $r$ is the current round, and $G$ is the set of nodes which have not been cluster-head in the last $1 / k$ rounds. Subsequently, each $\mathrm{CH}$ broadcasts an advertisement message and every regular node selects an appropriate $\mathrm{CH}$ for the current round. In the steady state phase, $\mathrm{CHs}$ gather data from other nodes, then aggregate and transmit it to the base station. LEACH needs every node to communicate with the base station through single-hop routing - something not feasible in most sensor networks. In addition, the selected $\mathrm{CHs}$ can be placed in the vicinity of network edges, causing the expenditure of much more energy by other nodes to send information to these CHs. To overcome this dilemma, the researchers have proposed LEACH-M [5] and LEACH-C [4], which are extensions of LEACH. LEACH-M forms multi-hop routing and LEACH-C centrally controls the base station in order to form CHs.

One of the algorithms which significantly outperforms LEACH is PEGASIS [6]. In PEGASIS, one node is selected from a chain of sensor nodes to be the leader node, which in turn transmits data to the base station after receiving transmissions for other nodes in the chain. The overhead caused by the dynamic formation of clusters in LEACH is reduced by PEGASIS. However, this accomplishment is countered by the lengthy delay introduced by the single chain for the distant node.

BCDCP [7] is a centralized cluster-based routing protocol. To select a CH in BCDCP, the base station forms a set of nodes with energy levels above the network's average energy value. Subsequently, this protocol groups the remaining nodes in one of the $\mathrm{CHs}$, after which the algorithm forms clusters via an iterative process until the desired number of clusters is achieved. Making use of $\mathrm{CHs}$ near the base station as intermediate nodes to form inter-cluster multi-hop routing in this protocol, leads to unbalanced energy consumption. In addition, communicating with the base station in each round results in high energy consumption and overhead.

To address this problem, Bejabar and Awan proposed an ADRP algorithm [8]. Rather than communicating with the base station at the end of each round for selecting $\mathrm{CHs}$, ADRP selects some nodes as future $\mathrm{CHs}$ for each cluster. Hence, at the end of each cycle, the role of the $\mathrm{CH}$ is switched to one of the future CHs without communicating with the base station. Although utilizing this method results in a significant decrease in energy used, inter-cluster single-hop routing and inefficient clustering in ADRP leads to un-even energy consumption, as well as energy holes in the network.

Still, none of the mentioned algorithms handles energy holes in the network, but Soro and Heinzelman have proposed an algorithm that does [9]. Their unequal algorithm divides network fields in cirques. Same-size clus- ters will be in the same cirque and there are various cirques of different cluster sizes. To ensure energy dissipation remains balanced, the $\mathrm{CH}$ is selected from a group of high-energy nodes to control network operations. In this algorithm, the CHs' positions must first be calculated to ensure that high-energy nodes are available to become CHs. EADUC [10] is an energy-aware protocol that uses unequally distributed clustering in heterogeneous multi-hop wireless sensor networks. CHs are selected based on the ratio of the node's residual energy and the average residual energy of neighbouring nodes. The irregular competition ranges are used as a basis to create uneven cluster sizes. CHs nearer to the base station will form smaller clusters as a way of preserving energy for routing between clusters. This algorithm suffers from extensive overhead caused by dynamic clustering.

A distributed unequal clustering algorithm called EEUC [11] elects CHs based on the nodes' residual energy. Every node will become a potential $\mathrm{CH}$ with a probability of $\mathrm{T}$. The uneven competition ranges are used by these tentative CHs for cluster formation of uneven sizes. As such, smaller clusters are closer to the base station (BS) than those further away from it. This way, a $\mathrm{CH}$ close to the BS is able to conserve energy for data forwarding between clusters. Even energy consumption between $\mathrm{CHs}$ is thus achieved. As $\mathrm{T}$ affects the quality of the generated CHs, some cases of T will have "isolate points" in EEUC.

\section{Network Model and Assumptions}

Since MDCR is an extension of ADRP, the same network model and assumptions as for ADRP have been used to develop this protocol. The assumptions are as follows:

1) The base station is far from the sensing field.

2) All the sensor nodes have a unique identifier (ID).

3) All of the sensor nodes are facilitated by power control.

4) Each sensor node is able to send information to any other node or to the base station.

5) There are no mobile nodes in the network.

6) Each sensor node can obtain location information via GPS.

The energy model has been adopted from [4], while the total cost of energy comes from Equations (2) and (3) where the transmitter and receiver transfer $r$ bit data messages over distance $r$ respectively.

$$
\begin{gathered}
E_{T}(k, r)=E_{T x}+E_{\text {amp }}(r) k, \\
E_{R}(r)=E_{R x} k .
\end{gathered}
$$

In Equation (2), $E_{T}(k, r)$ reflects the cost of total energy in the transmitter while $E_{R}(r)$ in Equation (3), shows the receiver's energy consumption. Parameters $E_{T X}$ and $E_{R x}$ in Equations (2) and (3) indicate the per-bit 
energy consumption for transmission and reception, respectively. The transmit amplifier requires energy to maintain an acceptable signal-to-noise ratio, ensuring reliable data transfer-shown as $E_{\text {amp }}(r)$. Finally, the energy for data aggregation is denoted by $E_{D A}$.

\section{The Orphan Nodes Problem}

Nearly all cluster-based routing protocols have orphan nodes. An orphan node is a node which does not belong to any cluster. A node may become orphaned for one of several reasons. For example, orphan nodes might be out of the CHs' communication range, and do not receive the advertised messages by $\mathrm{CHs}$ to join a cluster. Orphan nodes are of no use in the network for at least one round. Some solutions have been proposed in literature to make use of orphan nodes within the network. Firstly, it is possible to allow them to sleep in the current round so they do not consume energy; if in this situation, however, there happens to be some area in the sensing field which is not covered by any nodes in the respective round, network performance could be affected. Secondly, a protocol can be added, which lets the cluster member bring the orphan nodes into their clusters or allows them to communicate directly with the base station [12]. However, this method might increase the overall network's energy utilization. MDCR is able to deal with orphan nodes by using them as intermediate nodes to transmit data from $\mathrm{CHs}$ to the base station and form inter-cluster multi-hop routing. In this case, $\mathrm{CHs}$ close to the base station are able to preserve their energy for coordinating their clusters and energy consumption can balance out among CHs.

\section{MDCR Operation}

The whole MDCR operation is divided into rounds, each of which consists of initial and cycle phases just like ADRP. The initial phase is further divided into three stages: the partition stage, selection stage, and the multi-hop routing formation stage. The cycle phases are divided into the transmission and re-cluster stages. Figure 1 illustrates the MDCR procedure.

\subsection{Initial Phase}

Partition stage: Each sensor node transmits its energy level and location information to the base station. Global
Positioning System (GPS) can be used to obtain the sensor nodes' location. During the initial stage, this system is triggered and when all the information needed has been obtained, the base station selects the CHs. If a sensor node's energy level is above average in the network, it can be elected as a $\mathrm{CH}$. In addition, the MDCR places $\mathrm{CHs}$ into the centre of the clusters to distribute energy among the nodes. Once CHs have been selected, each of them broadcasts an Adv-Msg to inform the other nodes that it has been elected as the $\mathrm{CH}$ for the current cycle. Non-cluster heads pick the closest $\mathrm{CH}$ to them and send a Join-Msg. Sensor nodes that do not belong to any particular cluster will transmit an Orphan-Msg to the base station. In this case, the base station can identify orphan nodes and use them to form inter-cluster multi-hop routing.

Selection Stage: A $\mathrm{CH}$ has several responsibilities in the network. Therefore, the role of the $\mathrm{CH}$ should be rotated among the cluster's sensor nodes in order to distribute the energy load in the network.

During the selection stage, the base station elects the next heads. The nodes elected to be future $\mathrm{CHs}$ are called the next heads. MDCR reiterates the steps below to select the next heads:

1) The following equation is used by the base station to calculate the average energy of each cluster:

$$
T_{j}=\frac{1}{m} \sum_{i=1}^{m} E_{i}(t) .
$$

where $m$ is the number of sensor nodes in cluster $j$ and $E_{i}(t)$ is the node's current energy level.

2) Whichever sensor node's energy level is above $T_{j}$ - the threshold of cluster $j$-that is the one selected as a next-head candidate for the current round (Equation (5)).

$$
E_{i}(t) \geq T_{j}, \quad i \in C N H_{j},
$$

where $\mathrm{CNH}_{j}$ is the set of next-head candidate nodes for the current round in cluster $j$.

3) Once the $\mathrm{CNH}_{j}$ set is formed, the base station computes the distance between each member of $\mathrm{CNH}_{j}$ and $\mathrm{CH}_{j}(\mathrm{CH}$ of cluster $j$ ), and selects the nodes nearest to $\mathrm{CH}_{j}$. These nodes form a new set called $\mathrm{NH}_{j}$ (next heads of cluster $j$ ). In this case, the nodes near the center of each cluster become CHs during MDCR operation; so, CMs consume the same amount of energy to transmit their sensed data to $\mathrm{CH}$ and intra-cluster energy consumption is balanced.

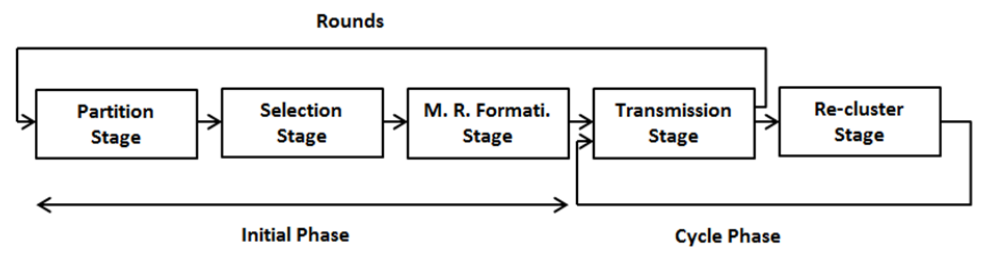

Figure 1. MDCR procedure. 
Four diverse types of sensor nodes are created at the end of the selection stage, as shown in Figure 2:

1) $\mathrm{CHs}$ which are responsible for gathering data, aggregating and transmitting it to the base station;

2) Next heads with the role of regular sensor nodes until the beginning of the re-cluster stage, one of which will be elected by the sensor nodes as a new $\mathrm{CH}$ for the coming cycle to switch to;

3) Regular sensor nodes that collect data from the surrounding environment to transmit to their $\mathrm{CH}$;

4) Orphan nodes which do not belong to any cluster and might be used as intermediate nodes to form intercluster multi-hop routing.

Multi-hop Routing Formation Stage: In this stage, base station assigns an orphan node to the $\mathrm{CH}$ and the set of the next heads which can form the shortest multi-hop routing path. After forming multi-hop routing, the base station sends a message to each sensor node. This message consists of $\mathrm{CH}$ and the next heads ID so that each sensor node is able to recognize the next head and switch to it at the end of each cycle.

\subsection{Cycle Phase}

Transmission Stage: Scheduling and data transmission are the two main activities in this stage. The regular node must be able to send its sensed data to the $\mathrm{CH}$ once a cluster has been formed. As such, the TDMA schedule is employed by MDCR. In TDMA, time is split into different slots which match the number of $\mathrm{CMs}$. $\mathrm{CH}$ assigns unique time slots to its members, in which the $\mathrm{CH}$ receives data sensed by its sensor node members. During data transmission activity, CMs send data to their respective CHs which will aggregate and transmit to the base station using inter-cluster multi-hop routing.

Re-cluster Stage: Again, in the multi-hop routing formation stage, each member in its respective cluster receives a message containing the cluster-head and next heads

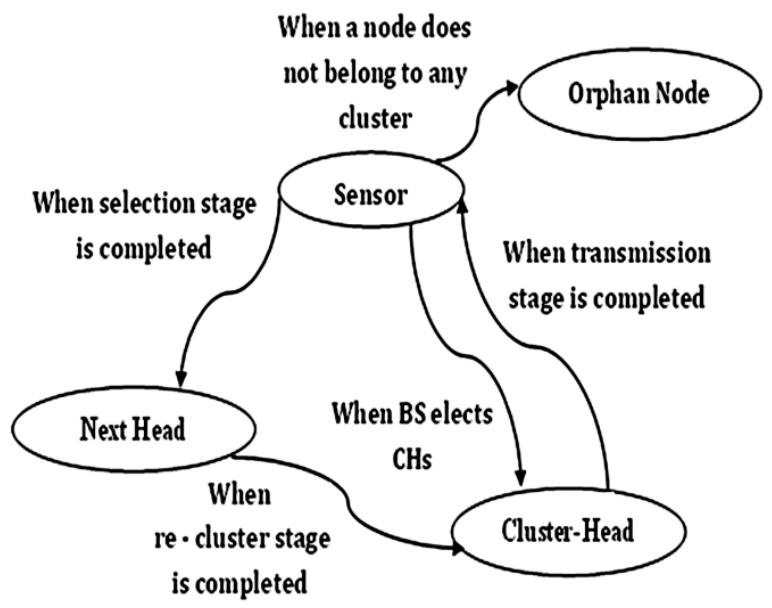

Figure 2. Node transition during MDCR operation.
ID from the base station. Therefore, the sensor nodes are not required to communicate with the base station for switching to the next head. At this point, the members of each cluster elect the first member of $\mathrm{NH}_{j}$ set as $\mathrm{CH}$ for the incoming cycle and switch to it. Once all sensor nodes have switched to the next heads, this stage is fulfilled and the transmission stage begins. The initial phase starts in case a next head is not accessible.

\section{Performance Evaluation}

We analyse the performance of MDCR in this section. The OMNET++ simulator [13] was used to simulate MDCR performance and compare it to the Adaptive Decentralized Re-cluster Protocol (ADRP), using the metrics shown below.

1) Network Lifetime: Network lifetime is defined by two metrics, namely a) first node dies and b) half of the nodes die due to battery depletion.

2) Total Energy Consumption: This test shows the energy used on the nodes in the network at certain time intervals, allowing us to see the protocol's energy consumption during operation.

3) Energy Consumption of CHs: This test shows the energy consumed by CHs at certain time intervals, allowing for the comparison of CHs' energy consumption in ADRP and MDCR. Simulation parameters are listed in Table 1.

\section{Simulation Results}

As pointed out in the previous section, two metrics are applied for estimating network lifetime: first, time until the first node dies and second, time until half of the nodes die due to battery depletion.

Figure 3 illustrates the time until the first node of the network dies over the number of cycles (a cycle is defined as the time it takes for the role of the $\mathrm{CH}$ to switch to the next head). With MDCR, all nodes are still alive for 88986 cycles; this number for ADRP is 80576 . Therefore, if network lifetime is defined as the time until the

Table 1. Simulation parameters.

\begin{tabular}{ccc}
\hline Meaning of parameter & Parameter value & Unit \\
\hline Network scale & $1000 \mathrm{~m} \times 1000 \mathrm{~m}$ & $\mathrm{~m}^{2}$ \\
Number of nodes & 100 & \\
Base station Location & $500 \mathrm{~m} \times 50 \mathrm{~m}$ & $\mathrm{~m}^{2}$ \\
Initial energy & 9.98 & $\mathrm{~J}$ \\
Data packet size & 1000 & $\mathrm{bit}$ \\
$E_{T \mathrm{Tx}}=E_{R x}$ & 50 & $\mathrm{~nJ} / \mathrm{bit}$ \\
$E_{\text {amp }}$ & 100 & $\mathrm{pJ} / \mathrm{bit} / \mathrm{m}^{2}$ \\
$E_{\mathrm{DA}}$ & 5 & $\mathrm{~nJ} / \mathrm{bit}$ \\
\hline
\end{tabular}


first network node dies, MDCR exceeds the ADRP network lifetime by $10.4 \%$. The reason for this improvement is the solution offered by MDCR for addressing inefficient clustering in ADRP. ADRP selects next heads without considering their location. Thus, it is possible for sensor nodes located at the edge of a cluster to get selected as next heads, and CMs consume high-energy loads to transmit information to them when they become $\mathrm{CHs}$. By employing the proposed solution explained in Section 5 , sensor nodes with sufficient energy and that are nearest to the cluster center are voted as next heads. So, members of the cluster consume approximately the same amount of energy to transmit information to the $\mathrm{CHs}$, thus prolonging node lifetime and subsequently, network lifetime.

Figure 4 shows the time it takes for half of the nodes in the network to die over the number of cycles. From this figure, it can be seen that it takes 94816 cycles for half of the MDCR nodes to fail due to battery depletion, while this number for ADRP is 86442. Similar to the previous metric, MDCR outperforms ADRP by $9.7 \%$.

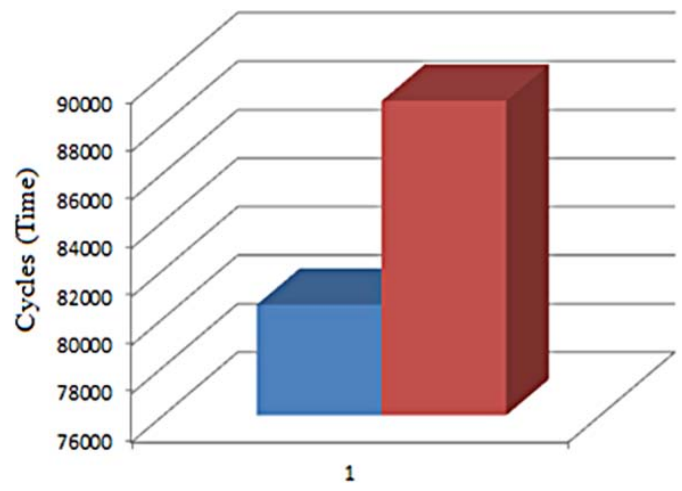

Figure 3. Time until first node dies.
Figures 3 and 4 illustrate that MDCR performs better than ADRP in increasing the network lifetime of all metrics and gains about $10 \%$ longer lifetime than ADRP.

Figure 5 illustrates the total energy consumption of the protocols over the number of cycles. Clearly, MDCR outperforms ADRP by $10.07 \%$. Using orphan nodes as intermediate nodes to form inter-cluster multi-hop routing is responsible for this improvement. ADRP applies inter-cluster single-hop routing which results in high energy consumption by $\mathrm{CHs}$ located far from the base station. Therefore, applying inter-cluster multi-hop routing by MDCR leads to a lack of balance in energy consumption among CHs and as a result, a decrease in total energy dissipation in the network. In addition, inefficient clustering in ADRP brings about high energy consumption of the sensor nodes located far from the CHs. To address this weakness pertaining to ADRP, the total MDCR energy consumption is decreased.

The final test comprises evaluating the energy dissipa-

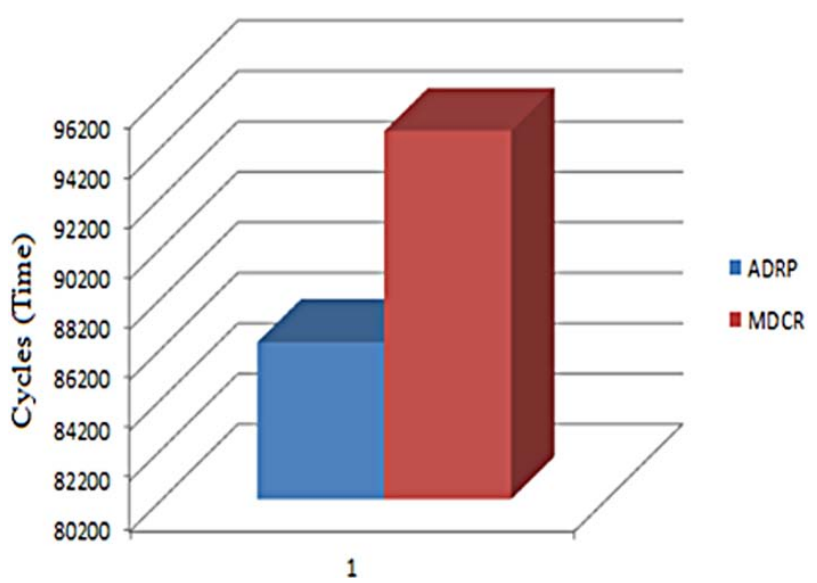

Figure 4. Time until half of the nodes die.

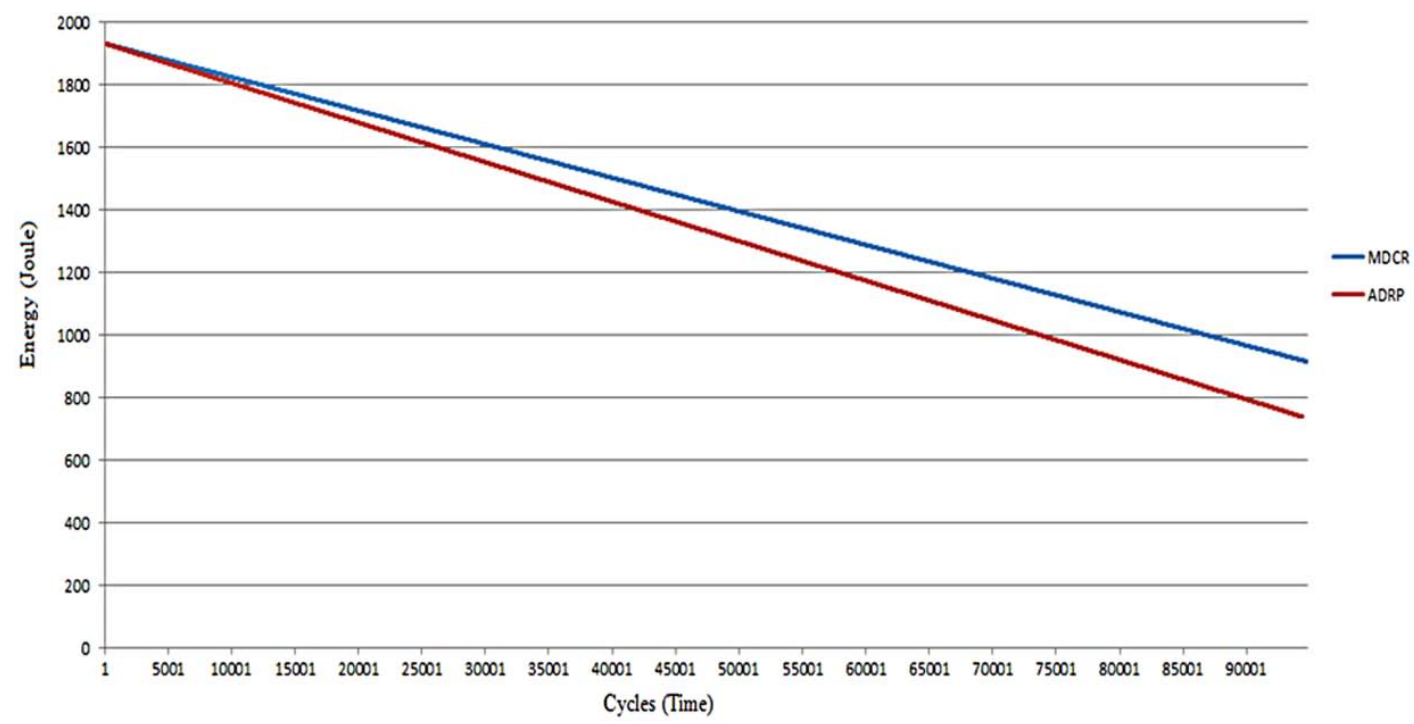

Figure 5. Total energy consumption. 
tion of CHs. Figure 6 illustrates the energy used up by $\mathrm{CHs}$ until half of the nodes die due to battery depletion. Obviously, the energy dissipation of CHs in MDCR is lower than that in ADRP. This improvement was achieved by using orphan nodes to apply inter-cluster multi-hop routing in MDCR. With this method, CHs located far from the base station consume less energy to transmit their information to the base station, while the closer ones are able to preserve some energy to coordinate their clusters.

\section{Conclusions}

In this paper, a Multi-Hop Decentralized Cluster-Based Routing protocol was proposed, which is an extension of ADRP. This algorithm utilizes orphan nodes in order to form inter-cluster multi-hop routing and tackle the energy-hole problem in the network. In addition, the sensor nodes' location is considered as a metric for selecting next heads, something that would solve the problem of inefficient clustering in ADRP.

Simulation results indicate that compared to ADRP, the performance of the proposed protocol is superior in terms of network lifetime, total energy consumption, and energy consumed by CHs.

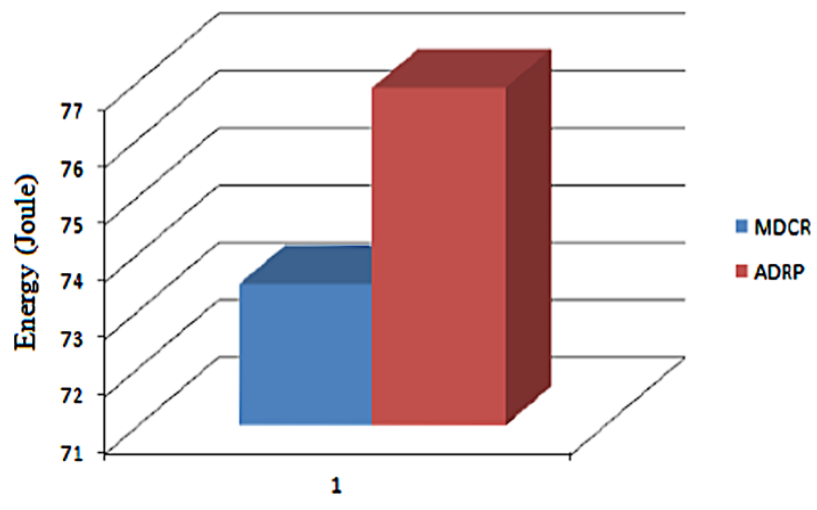

Figure 6. Energy consumption of cluster-heads.

\section{REFERENCES}

[1] I. F. Akyildiz, W. Su, Y. Sankarasubramaniam and E. Cayirci, "A Survey on Sensor Networks," IEEE Communications Magazine, Vol. 40, No. 8, 2002, pp. 102-114. http://dx.doi.org/10.1109/MCOM.2002.1024422

[2] K. Akkaya and M. Younisc, "A Survey on Routing Protocols for Wireless Sensor Networks,” Ad Hoc Networks, Vol. 3, No. 3, 2005, pp. 325-349.

http://dx.doi.org/10.1016/j.adhoc.2003.09.010
[3] N. Aslam, W. Phillips, W. Robertson and S. Sivakumar, "A Multi-Criterion Optimization Technique for Energy Efficient Cluster Formation in Wireless Sensor Networks," Information Fusion, Vol. 12, No. 3, 2011, pp. 202-212. http://dx.doi.org/10.1016/j.inffus.2009.12.005

[4] W. B. Heinzelman, A. P. Chandrakasan and H. Balakrishnan, "An Application-specific Protocol Architecture for Wireless Microsensor Networks, " IEEE Transactions on Wireless Communications, Vol. 1, No. 4, 2002, pp. 660670. http://dx.doi.org/10.1109/TWC.2002.804190

[5] Y. Li, X. H. Zhang and Y. Z. Li, "Algorithm of Cluster Head Multi-Hops Based on LEACH,” Computer Engineering and Design, Vol. 28, No. 17, 2008, pp. 41584160 .

[6] S. Lindsey and C. Raghavendra, "PEGASIS: Power-Efficient Gathering in Sensor Information Systems," Proceeding of the International Conference of the IEEE Aerospace, Big Sky, 9-16 March 2002, pp. 1125-1130. http://dx.doi.org/ 10.1109/AERO.2002.1035242

[7] S. D. Muruganathan, D. C. F. Ma, R. I. Bhasin and A. O. Fapojuwo, "A Centralized Energy-efficient Routing Protocol for Wireless Sensor Networks,” IEEE Communications Magazine, Vol. 43, No. 3, 2005, pp. 8-13. http://dx.doi.org/ 10.1109/MCOM.2005.1404592

[8] F. Bajaber and I. Awan, "Adaptive Decentralized ReClustering Protocol for Wireless Sensor Networks," Journal of Computer and System Sciences, Vol. 77, No. 2, 2011, pp. 282-292. http://dx.doi.org/10.1016/j.jcss.2010.01.007

[9] S. Soro and W. B. Heinzelman, "Prolonging the Lifetime of Wireless Sensor Networks via Unequal Clustering," Proceedings of the 19th International Conferences of the IEEE IPDPS, Colorado, 4-8 April 2005, pp. 236-243. http://dx.doi.org/10.1109/IPDPS.2005.365

[10] J. Yu, Y. Qi, G. Wang, Q. Guo and X. Gu, “An Energy-aware Distributed Unequal Clustering Protocol for Wireless Sensor Networks," International Journal of Distributed Sensor Networks, Vol. 2011, 2011, Article ID: 202145. http://dx.doi.org/10.1155/2011/202145

[11] C. F. Li, M. Ye, G. H. Chen and J. Wu, “An EnergyEfficient Unequal Clustering Mechanism for Wireless Sensor Networks," Proceedings of the International Conference of the IEEE on Mobile Adhoc and Sensor Systems, Washington, 7 November 2005, pp. 1-8. http://dx.doi.org/10.1109/MAHSS.2005.1542849

[12] L. B. Oliveira, et al., "SecLEACH on the Security of Clustered Sensor Networks,” Signal Processing, Vol. 87, No. 12, 2007, pp. 2882-2895. http://dx.doi.org/10.1016/j.sigpro.2007.05.016

[13] A. Verga, "OMNeT++ Discrete Event Simulation System Version 4.2 User Manual,” 2011. http://www.omnetpp.org/doc/omnetpp/Manual.pdf 